
Abstracted by Edward F. Malone, author. University of
Cincinnati.

Sharpening microtome knives.

This article discusses the nature of the edge of knives and the action of abrasive substances under different conditions. The preparation is described of hones sufficiently large to permit the knife to be sharpened without any portion projecting beyond the edge of the hone. The surface of the hone is leather glued to stone; the leather is then finished plane and treated with castor oil and grit. The methods of refining and testing grit are described at length, together with the proper care and use of the hones. Types of suitable honing backs are described and illustrated. The double concave knife is recommended, together with a large knife-holder adjustable for tilt. The method described is suited to the production of an edge uniformly good throughout its entire length rather than one of the very highest perfection. 
ACTHOR'S ABSTRACT OF THIS PAPER ISSUED

BY THE BIBLIOGRAPHIC SERVICE, SEPTEMBER 11

\title{
SHARPENING MICROTOME KNIVES
}

\author{
EDWARD F. MALONE
}

Anatomical Laboratory of the University of Cincinnati

FIVE FIGURES

In this article I propose to discuss the imperfections in design of microtome knives, the methods of making faulty knives serviceable, and especially the principles and methods of sharpening such knives as permit of being sharpened on a hone. That the edge of knives is usually unsatisfactory is a fact that permits of no discussion; the expression of more general complaint is due not to the absence of dissatisfaction, but to the belief that a good edge can be obtained only by an expert. While this belief is in general correct, the services of an expert are necessary only because he sharpens imperfect knives by methods requiring great skill. I propose to describe a method by which an excellent edge may be obtained without the use of great skill and which demands only the exercise of care and intelligence. The edge so obtained is not of the highest refinement, but is characterized by uniform excellence throughout its entire length.

The edge of a knife is formed by the intersection of two narrow plane surfaces, the cutting-facets; the base of the included wedge is continuous with the body, and each facet with a surface of the knife. The facets result through the formation by the hone of fine oblique parallel scratches, and these scratches result in a serration of the edge with the teeth directed towards the heel of the knife. To facilitate rapid cutting, the knife rests upon the hone only on its narrow cutting-facet and a second broader facet situated on the surface of the knife immediately adjoining the back; both of these facets or supporting surfaces lie in the same plane. To insure the knife's resting upon these two surfaces of limited area, each side is ground concave or else the back is elevated by the attachment of a special honing back. 
In the process of sharpening it is thus evident that the edge is not formed directly, but is the product of the formation and development of the two cutting-facets, and that any defect which the edge possesses is the expression of some defect in one or both of the two intersecting planes by which the edge is formed. Since the cutting-facets are not smooth surfaces, but are marked by
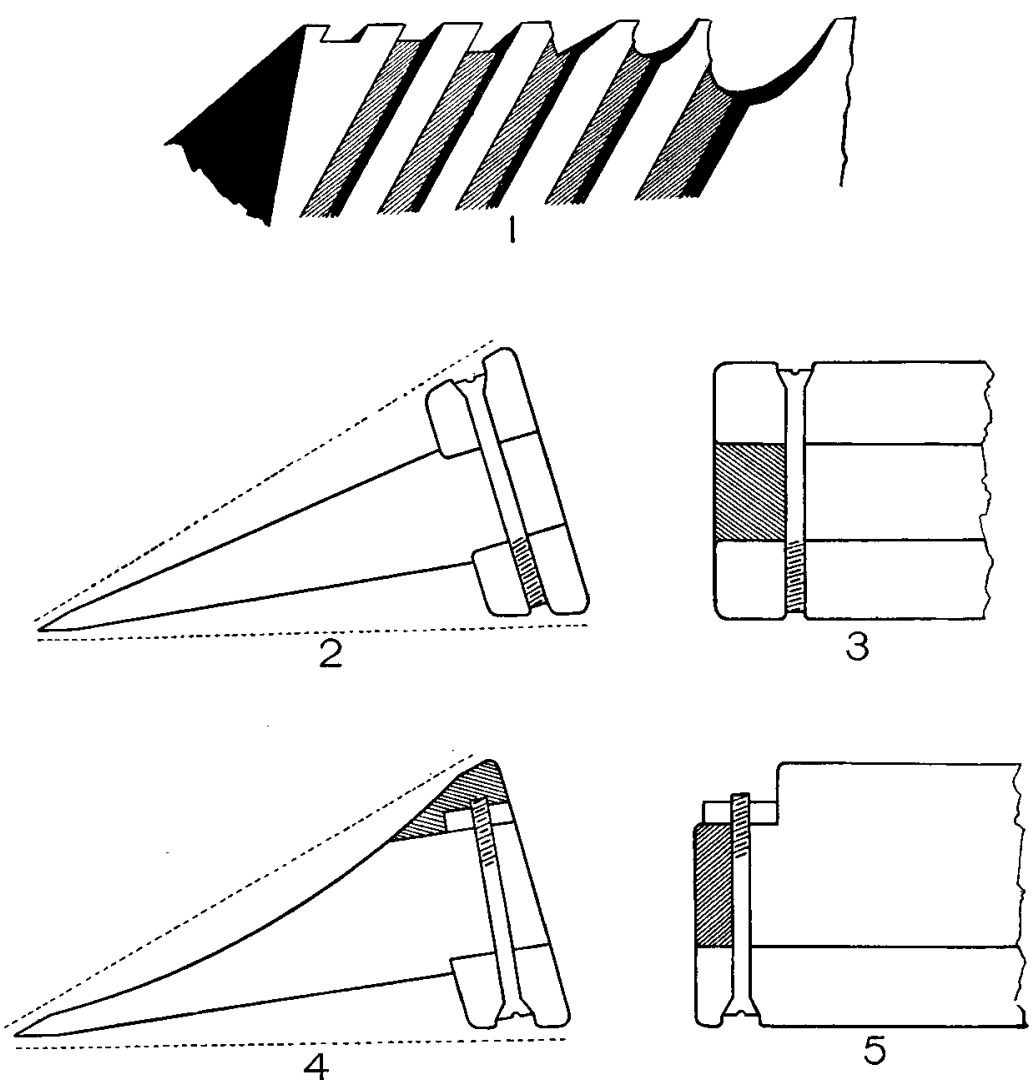

Fig. 1 Theoretical diagram drawn from wood model. From left to right a scratch intersecting with (1) near facet, (2) far facet, and (3) with another scratch; also scratches resulting in (4) a small V-shaped break, (5) a small U-shaped break, and (6) a true gap.

Figs. 2 and 3 Wedge-shaped knife and back. Shaded region in figure 3 represents slot; note rounded corners.

Figs. 4 and 5 Planoconcave knife and back. Shaded region in figure 5 represents slot. 
oblique, parallel scratches, it follows that they cannot intersect in a smooth linear edge. The scratches upon both facets do not necessarily occur opposite one another and often cut the surface of the opposite facet after crossing the median plane. Since the scratches are parallel to the surface of the facets, their intersection with one another or with the opposite facet is at the same angle as that of the elevated portions of the facets. It is then evident that since it is impossible to form two perfectly plane surfaces intersecting to form a linear edge one should use every effort to approach this condition as completely as possible, to reduce the size of the scratches to a minimum while retaining the generally plane facets, without which a rounded edge results. An important reason for uniformly fine scratches is that when a deep one occurs the local pressure on the facet is tremendously increased, resulting not merely in a deeper serration, but also in an irregular breaking away of the steel near the edge which further deepens the gap (fig. 1).

The question arises as to why it is impossible to form two plane facets, polished free from even the finest scratches and intersecting in an edge free from serrations. Theoretically, this is possible by suitable stropping, but practically the lessening of the serrations is due largely to direct rounding off of the edge rather than to the primary removal of the scratches to which the serrations are due. In addition to the tendency to round off the edge, the strop is a source of further serious injury to the knife, the nature of which will be considered later.

Intersecting plane cutting-facets may be formed by means of a fine wheel or a fine plane hone. The wheel cuts the knife on the cutting-facet alone, while the plane hone cuts in addition a second facet adjoining the back of the knife. Since this second facet lies in a continuation of the plane of the cutting-facet, these two surfaces will stabilize the knife, and however often it be sharpened on a plane hone the angle and line of intersection of the cuttingfacets will necessarily remain constant. Of course a knife could be thus ground on a wheel by passing the knife in a fixed plane tangent to the periphery of the rotating wheel, and the sharpening could be continued without interruption on a plane hone, since 
a knife so ground would have on each side two plane facets lying in the same plane. It is hard to see why knives should not be so ground, but as a matter of fact they seldom are; usually the cutting-facets are ground without relation to the back, and this is unsatisfactory because the new edge is not sufficiently keen and cannot be improved on a plane hone and when dull must be reground on a wheel. This independence of cutting-facet and back is such that when the edge is placed in contact with a plane surface only one corner of the back will rest on that surface, the other corner failing to touch in many cases by as much as several millimeters; on the opposite side of the knife the opposite corner of the back is apt to fail to touch. Such a knife must be reshaped by grinding, and after the cutting-facets have been developed on a plane hone together with the secondary supporting facets, the cutting-facets should be narrowed by concave grinding. Of course a knife should be in this condition when purchased, but since they are usually not to be obtained in this condition they should be sent to be properly finished.

Accordingly, although the scratches made by a fine wheel are too coarse and cannot be reduced on a plane hone, the wheel is of great use in generally reshaping a knife, in removing large gaps, and in narrowing cutting-facets through concave grinding. The value of the wheel for this work lies in the uniformity and rapidity with which it cuts. On the other hand, the cuttingfacets must be formed on a plane hone and the rate of cutting must be exceedingly slow, since a very coarse hone or the use of any external pressure will cause deep scratches and gaps. Since the steel must be removed very slowly by means of minute and uniform scratches on the cutting-facets, the process can be shortened only by keeping these facets narrow, thus reducing the amount of steel to be removed. By keeping the facets narrow a minute amount of steel can be delicately yet rapidly removed, and the keenness of the edge restored by a few strokes; theoretically to employ wide facets means lengthening the work from minutes to hours or even days, but practically it means failure, for in such a prolonged process accidents are almost sure to occur, thus indefinitely postponing completion. But there is a minimum 
width of cutting-facet below which insufficient support is furnished, resulting in a rounded or broken edge. This minimum width depends upon the total pressure exerted upon that portion of the cutting-facet in contact at any instant with the hone, and since all external pressure must be avoided one needs only to consider pressure due to the weight of the knife. The most important factor is the angle formed by the cutting-facets, for as the back is elevated a greater proportion of the weight falls upon the cuttingfacets. A thick, broad knife weighs more per unit of length, but this is counteracted by the shifting backwards of the center of gravity. A long knife on a narrow hone will be supported by less than the total surface of the cutting-facet, and even if the hone is so large as theoretically to support the entire surface of the facet, yet due to inequalities in the surface of the hone a long knife will exert greater local pressure than a short one, and coarse scratches with resulting gaps are always due to excessive local pressure. Practically I recommend a cutting-facet about $1 / 2 \mathrm{~mm}$. in width, with a minimum width of $1 / 4 \mathrm{~mm}$. and a maximum of $1 \mathrm{~mm}$.; when the width exceeds the latter measurement, the cutting-facet should be narrowed to about one-half millimeter by removing its rear portion through concave grinding.

Narrow cutting-facets are obtained either by a double-concave grinding or, in the case of a wedge-shaped knife, by elevating the back through attaching a special honing back. The latter type of knife permits the facets to widen more rapidly than in the case of the double-concave knife and presents other difficulties to be considered later. The wedge-shaped knife is by some supposed to be more rigid than the double-concave type, but one should not overlook the fact that the wedge-shaped knife with attached honing back is usually sharpened at a more obtuse angle; to obtain the same angle of edge in knives of the same width the back of the double-concave knife should have the same thickness as that of the other type with its honing back attached. The rigidity of a knife depends almost exclusively upon the angle of the edge, which should be a little more obtuse than is required by the work which the knife has to do, and with equal angle of edge there can 
be no essential difference in rigidity between a thin wedge-shaped knife and a thick wedge-shaped knife subsequently grounå concave. Moreover, in comparing types of knives one is apt to overlook not only the angle of the edge, but those manipulations of the knife by which its tilt and slant are adjusted to the thickness of the section and the nature of the tissue. The only knife-holder worthy of consideration is one adjustable for tilt and large enough to hold a thick knife; the unfortunate replacement of the complete knife by the thin knife with removable back is encouraged by the small knife-holders usually supplied. While it may be possible that for certain kinds of work a plane surface is superior to one which is concave, yet this possible advantage can never compensate for an inferior edge, and a good edge is more easily obtained on a double-concave knife. Bearing in mind the fact that a doubleconcave knife should be ground from a wedge of steel whose angle, and therefore the angle of the finished edge, is suitable for the work required of it, I unhesitatingly recommend this type of knife. And I believe that the objections to it depend in large measure, if not entirely, upon the use of an edge of unsuitable angle and upon improper adjustment of the knife.

When the edge of a knife is too acute, the only way to increase its angle is to employ a special honing back. The honing backs in the market are useless, since they fail to stabilize the knife when the latter rests upon a cutting-facet, often shift position during honing, and cannot be replaced on the knife in the same position. I have had much experience with them and am convinced that those on the market are failures. This inferiority of design and manufacture of honing backs is not the fault of the manufacturer, but is due to the unexacting demands of their customers who have never considered honing in the light of a rational procedure, but one so closely allied to the supernatural as not to exclude the hope of obtaining a good edge by the formation of two rounded shifting facets determined by the unstable back. Moreover, these backs are supposed to fit any knife of a certain size and type, regardless of the individual peculiarities of knives due to warping, and often a back is not reserved for one particular knife, but is used on several. Until a more discriminating demand arises, 
one cannot expect manufacturers to regrind their knives true after tempering and to supply each perfectly symmetrical knife with a honing back which will fit perfectly and can always be attached in exactly the same position. In fact, I recommend special honing backs only for expensive knives which one happens to possess and which cannot otherwise be suitably sharpened. No honing back can make a knife equal to a double-concave knife, and accordingly the latter is the only type which should be purchased. A honing back should not be attached to a double-concave knife; if the edge of such a knife is too acute, save the knife for suitable tissue; for using an oblique stroke, the increased acuteness of the effective angle of the edge will lessen the danger of injury, and under these conditions such a knife may be ideal. The two types of knives which must have honing backs are the wedge-shaped and plano-concave; the former should have an elevating strip on each side and the latter on the plane side only.

In connection with honing backs the following principles should be observed (figs. 2 to 5 ). Those portions of the knife and elevating strips which lie in contact should be ground perfectly plane and each strip should rest against a slight shoulder on the knife; this insures a perfect fit necessary for stability and accuracy in reassembling. The two surfaces of the knife in contact with the strips are best ground parallel to one another to insure accuracy in drilling the strips as they can be drilled while clamped together. The knife cannot be drilled and therefore the screws are made to lie in narrow slots, at each end of the knife, ground perpendicular to its two parallel surfaces; if a third screw seems advisable, a slot could be ground for it in the middle of the back of the knife. The elevating strips must be drilled and tapped and therefore of soft steel or iron, the tendency to wear away rapidly being balanced by the relatively large surface. These strips should be of sufficient thickness to give, after they are reground, the desired angle. Due to the grinding away of the surfaces of the knife, these strips are thick enough to avoid vibration and bending and to allow countersinking of the machine screws. The machine screws should have a diameter of $1 / 8$ inch and be flat-headed, since this type can be more accurately countersunk. The slots 
should be so deep that the head of the screw will be removed from the end of the knife by at least $1 \mathrm{~mm}$. When attached the elevating strips should be so ground that their surfaces lie in the same plane as the edge, and after the formation on a hone of the cutting-facets the latter should be narrowed by lightly grinding away their posterior border. All edges and corners are rounded off to protect the hone. Of course each knife must have its own back and each should be identified by number, and the pieces marked to insure proper assembling. In case of the planoconcave knife, the back of the concave side is ground parallel to the plane surface, but only at each end. While I have as yet never had the equipment to make such a back, I have prepared much cruder ones which are satisfactory, and accordingly do not hesitate to recommend the back described above. As previously stated, such a back will often make an expensive knife usable, but in ordering new knives double-concave knives of proper angle should be specified.

Assuming that we have a double-concave knife or a knife with a honing back which remains rigidly in place and can always be replaced in exactly the same position, we may now consider hones and methods of sharpening. From the nature of the cuttingfacets it follows that a hone should be plane and should remain so, and that if it is large enough to permit one to keep the entire knife always upon the hone the process of keeping the facets plane will be made more accurate and much easier to accomplish. For if the hone is plane and cuts finely and if the entire length of the facet rests upon the hone little skill is required to produce a good edge. Assuming the size of the desired hone to be 1 foot by 2 , it is evidently impossible to secure a hone of the usual type in this size, and artificial abrasives which can be made into a stone are too coarse.

At first I tried sharpening on plate glass with fine carborundum powder and water, and after several years found why this method can never be successful. The facets appear very evenly and finely frosted, but partial polishing reveals the presence of deep scratches and numerous pits extending deep into the steel. Unless of extreme fineness a freely moveable grit between two moving 
hard surfaces will always cause the formation of pits and gashes under cover of the delicate surface finish. This method has been successfully used by Funck (Zeit. f. wiss. Mik., 1910), who employs an extremely fine grit, thus limiting the method to the formation of the final edge; however, the danger from the accidental presence of even relatively fine foreign grit is always present. Reflecting upon the difference in behavior between a movable grit and the fixed grit of a stone, it occurred to me that the complete fixation of the grit was far from ideal; for instance, the tendency of the stone to pull out particles of steel and the danger of injuring the edge on the hard stone in case of a slight accident. As a result I decided to try the effect of partial fixation of the grit, embedding it in a surface which would not hold it firmly against excessive resistance, and covering the surface with a thin layer of grit which would pack into a self-leveling abrasive surface unyielding under the weight of the knife. The material selected was leather treated with castor oil and has justified all expectations.

The leather surface must have a rigid support which will not warp, and accordingly wood cannot be used. I use any soft stone which can be easily cut with a saw, ground plane, and the corners and edges finished with a rasp, while sufficiently porous to hold glue. The upper surface of the stone is ground plane after well rounding off the corners and edges to prevent breaking. Three such stones should be prepared, since a course, a medium, and a fine hone are necessary. The two finer hones should measure 8 inches by 24 , while the coarse hone should measure 12 by 24 inches. Before handling the leather all grit must be removed from the stone, hands, clothing, and surroundings, since any grit once embedded in leather can never be removed. For leather I use a good grade of cowhide of uniform thickness and texture. In clean surroundings the leather is cut to the proper size, leaving a margin of stone at least $1 \mathrm{~cm}$. wide and the corners of the leather cut off round to correspond to those of the stone, since sharp corners tend to become detached. A plane surface is selected of suitable size and such a nature as to permit the use of large clamps, and is covered with a piece of clean paper to prevent 
the leather from sticking to the surface beneath the paper. The leather is placed dressed side down upon another piece of paper in a different location and both the leather and the surface of stone given a coat of hot glue of a good grade; from now on one must work quickly. The two coated surfaces are placed in contact and the leather smoothed out, pressing out firmly the excess of glue which is wiped off with a damp cloth, care being taken to remove any glue on the upper surface of the leather. The hone is then placed carefully, leather surface down, on the paper-covered surface prepared for it and the clamps carefully and uniformly tightened to counteract the tendency of the leather to shift its position.

After twenty-four hours the clamps are removed and the leather surface carefully dressed with a plane. The plane should be at least 15 inches in length, very sharp and free from gaps. The plane must not cut deeply, since this might cause the leather to tear or crack, and should be moved in all directions, especially along the diagonals, frequently turning the hone end for end, so as to insure a plane surface. When the surface is plane the edges and corners are slightly beveled and the surface finished by lightly shaving with a sharp microtome knife. This knife must lie flat on a plane surface and is lightly moved edge first in different directions in such a manner as to render the surface plane and evenly finished. The leather surface should have a fine velvet finish and is now ready for the oil and grit. When not in use each hone should be covered with its own properly labeled dust-proof top of wood or cardboard.

The leather surface of each hone is treated with castor oil, which should be uniformly distributed and thoroughly rubbed in. More oil is added and upon the addition of the appropriate grit the resulting paste is rubbed into the leather; fresh grit is added from time to time. The rubbing should be vigorous and rags or paper should be used. As the paste becomes thick the velvet surface of the leather begins to come away with the paste and the leather becomes smoother and firmer. This thick paste fills the pores of the leather, and when the hone is in use will form a thin firm layer over the leather which should be covered in turn by a 
very thin layer of dry grit. Do not add too much oil, since the excess must be removed and this is tedious and requires the use of much grit. After a day or two when the oil has soaked in the excess of oil is removed from the leather by repeated and vigorous rubbing with many clean pieces of paper toweling. This process should be thoroughly carried out so as to avoid the necessity of too frequent repetition. Before use the leather surface is brushed off, rubbed with the palm of the hand to free it from foreign particles, and a little grit added, which is rubbed in by hand and smoothed with the palm. Beginning from the time that the grit is first rubbed into the hone, each hone should rest on a separate table in surroundings free from coarse grit and should have its own dust-proof top. Since the surface is always smoothed with the palm, the hands and finger-nails must be carefully cleaned, especially when one is to use the finer hones, and one should always work with bare forearms. Honing backs must be removed from knives and each carefully washed with soap and water and then dried, paying especial attention to recesses in which grit may collect; this is one of the disadvantages of such backs. An ideal source of contamination is the greasy castor oil bottle and its cork, which should be protected from all grit and carefully cleaned before using. The necessity of care in protecting the fine hones is imperative, since coarser grit once imbedded can never be removed; it can, however, by repeated cleaning be rubbed into the leather and kept covered with fine grit.

The nature of the surface of the leather hones must be carefully considered; the surfaces of the three hones differ only in that the finer the grit the more delicately it cuts and the more readily it packs into a compact layer. The addition of castor oil to the leather changes the hard leather with superficial velvet finish, a finish which would round off the edge, into a dense, sticky, homogeneous mass. Thus the velvet surface is either rubbed off or else incorporated with the rest of the leather, and the softened pliable leather is more receptive to the grit. Without the presence of grit oiled leather will cause a knife to adhere tightly and is absolutely useless. This tendency to cause the knife to adhere continues to reappear to a slight extent after the grit is added, 
and when slight may be overcome by rubbing up the grit and redistributing it with the palm of the hand. But in new hones frequent rubbing off of the excess of oil with paper is necessary. The carborundum grit fills up the pores of the leather and renders it firmer, and while rouge on oiled leather will cause the knife to adhere hopelessly, carborundum grit if properly manipulated will form a surface over which the knife glides without any pressure other than that due to its own weight. The dry grit should form, above the firm paste, a definite layer, but one whose thickness is almost inappreciable. When gently roughened up with the palm, the grit, as the knife passes over it back foremost, fills in any irregularities in the leather and automatically forms a plane surface. The leather is inelastic, but is readily dented through pressure exerted by a sharp object, such as a corner of the knife or a finger-nail. These marks may be removed by rubbing or forcibly ironed out by any object having a straight rounded edge, such as the edge formed by the back and main surface of the knife, but such depressions are harmless unless excessive and soon become filled with dense paste or dry grit.

This soft, homogeneous consistency of the leather is the source of advantages not found in case of a stone surface. Under the weight of the knife the leather is absolutely unyielding, the surface being as dense as the rest of the leather, and in this respect it is equal to a stone surface, while in other respects it is superior. The leather does not cause the knife to vibrate, it always remains plane, and in case of slight accidents the soft leather will not hold the grit firmly, thus avoiding great injury to the knife. Such accidents as slightly elevating the back of the knife, the presence of foreign particles on the hone, or striking the edge of the knife against the edge of the hone, while very serious in case of a stone surface, cause relatively little damage in case of the leather hone. In other words, oiled leather is as harmless a substance as will support a knife without yielding under its weight, while the particles of embedded grit are capable merely of individual action which is limited, in the event of unduly increased local or general pressure, by the ability of the particles of grit to give way; whereas a stone acts both through its rigidly fixed 
individual particles and also through the unyielding mass which these particles form. Moreover, unlike stone or plate-glass, leather cannot give off any hard particles such as are a source of constant danger in using a hard surface. The ease and certainty with which a good edge is obtained on a leather hone is the result of avoiding or reducing to a minimum the effects of those frequent slight accidents which are often undetected.

The nature of the grit and the method of refining it will now be considered. The entire support of the cutting-facet of the knife is equal to the combined support of all the individual particles of grit with which the facet is in contact. In the case of coarse grit, the tops of these particles are relatively far apart, thus offering relatively less total support to the facet and thereby causing the facet to exert a relatively greater local pressure on each particle; moreover, the greater height of these particles permits them to cut more deeply into the facet before the latter receives additional support from deeper-lying particles. In the case of a fine grit, the total area of contact with the facet is greater while this increased surface of contact is formed by a much larger number of particles, and consequently the local pressure on each particle is decreased, thus causing them to cut less deeply. Grit cuts most rapidly when used upon a hard surface, such as a plate of glass, less rapidly when held together by a binding substance, as in the case of a stone (except when particles of grit become dislodged), and least rapidly when used upon a surface which will allow the particles to sink in, thus tending to equalize local differences in pressure. A large particle of grit will cause much less damage when used with other particles of the same size than when used with finer particles, since in the latter case the entire support of the facet is furnished by the single large particle which extends above the general surface of the fine grit; moreover, the fine grit will form a delicate edge so that under these conditions a large particle will not only cause a deeper scratch, but will break away the edge to a greater extent. Isolated coarse scratches with their accompanying gaps are merely the result of abnormal local pressure, of local rapid cutting during a stage of honing wherein the general rate is slow; whereas during a stage of honing character- 
ized by general rapid cutting the roughness of the edge must be considered normal. Accordingly, during any stage of honing the size of the scratches should be nearly constant, larger or smaller according to the rate at which the steel is removed. In other words, however coarse or fine the grit, it will cut with maximum rapidity and with minimum injury when its particles are of uniform size.

Carborundum grit should be used, since in addition to its extreme hardness it spreads into a smooth layer without the tendency to form isolated lumps; a 5-pound can of ' $\mathrm{FF}$ ' and also of the 'Sixty minute' powder should be procured. It is absolutely essential to refine these commercial powders, and when refined the coarse or FF powder is used for the coarse hone, while the Sixty minute yields, after grinding it into a finer powder, two grades of powders for the medium and fine hones, respectively. The refining is carried out by settling in water and the result controlled under the microscope with the aid of a micrometer eyepiece. Under each of two conditions some of the grit will fail to settle in water, for dry grit will form a surface film over the water and air bubbles will carry a film of grit upwards; these circumstances, while of no importance during the elimination of particles too small to be of use, involved most serious consequences when the particles to be eliminated are too coarse for use. Accordingly, a little of the dry powder should be placed in a mortar, and when thoroughly mixed with water it is poured into a liter measuring cup or pitcher, which when filled is caused to overflow by the addition of water and the floating grit and air bubbles brushed off. During subsequent operations, whenever coarse grit is to be eliminated, the bottles should be filled to overflowing immediately before allowing contents to settle, and the surface grit floated off before introducing the siphon; moreover, as the surface falls in the bottle it may regain a film of grit from that adherent to the sides so that the upper centimeter of the contents should never be siphoned off. One must remember that the position of a particle of grit which has been allowed to settle for a definite time depends not only upon its weight, but also upon the point from which it started; therefore the separation can be 
accomplished only by repeated settling and siphoning with the use of many changes of water. All particles above a certain size will settle each time, but will be mixed with many useful smaller particles, and after all the latter have been recovered by repeated settling all the useful particles can be made to settle each time, but must be washed free by repeated settling from those particles which are too small to use. Another source of contamination is due to the disturbing of the settled grit through the current set up by the siphon, so the end of the tube should never closely approach the layer of settled grit and the stronger the stream the greater should be the distance; this is naturally of greater importance in the case of the finer grades. Finally the grit should be dried at room temperature, since if dried over a flame it will unite into dense masses which will not disintegrate upon rubbing.

The FF carborundum powder contains relatively few particles too coarse to use upon the coarse hone, but many which are too fine, including a considerable amount of what appears to be rouge or else mud; on account of this contamination the finer particles should not be saved for the finer hones. In preparing the grit for the coarse hone I reject all particles large enough to settle 10 or 12 inches in one-half minute, and reject all those too small to settle 5 or 6 inches in one minute. It is best to begin by removing the excessively small particles by thoroughly rubbing up the grit in a liter cup of water, allowing it to settle one minute, and pouring off half the contents; this is repeated until the water comes off clear. After this preliminary cleansing, the grit is poured into a bottle 12 or 15 inches high holding at least a gallon, and by repeated settling all grit siphoned off except that too coarse to use. Each time the contents is thoroughly mixed without undue formation of bubbles, the bottle filled to overflowing and the contents allowed to settle for one-half minute, after which the surface layer of grit and bubbles is again brushed off and the rubber tube introduced so that its inlet reaches within about 3 inches of the bottom. The contents is siphoned into a second gallon bottle, and as the pressure falls the inlet can be lowered, but never nearer to the settled grit than 1 inch. Since 
the suspension is opaque, the tube should be so marked by adhesive tape that one can tell the position of its inlet; after each operation the tube should be washed inside and out. The above operation is repeated until all useful grit is removed from the first bottle, and since it is convenient to collect all of this useful grit in one bottle, each time this second bottle is filled its contents is mixed, allowed to settle two minutes, and siphoned off in the sink. After all the useful grit has been collected in the second bottle, the smaller particles must be eliminated by allowing to settle one minute and siphoning off into sink the upper half of contents, keeping inlet of siphon at a depth of 5 or 6 inches; after mixing the rest of contents and settling for the same time, the rest of the suspension is siphoned off. This is repeated until the water comes away clear. The grit should be well mixed and a sample examined under the microscope. The average size of particles should be 35 to $40 \mu$; there should be few smaller than $15 \mu$, although some as small as $2 \mu$ will appear. Particles $75 \mu$ in length should not measure over 50 in width, but a few narrow ones may occur with a length of $150 \mu$. Since the particles are separated according to weight and since their shape varies, it is impossible to prevent a considerable variation in their long diameter. In this grade of grit the danger is in the presence of too many fine particles below 15 or $20 \mu$ in diameter. After testing, the grit is filtered, washed with distilled water and preferably alcohol, and spread out to dry without the use of heat. It is kept in a properly labeled bottle with a cork stopper; a glass stopper must not be used.

The Sixty minute carborundum powder from which the medium and fine grades of grit are prepared is much cleaner than the FF powder and contains few particles too small to use, while the majority have to be ground still smaller. At first it is best to remove from the powder all particles small enough to use and then grind up the larger ones, since grinding is liable to eliminate too many of the size suitable for the medium grade of grit. The dry grit should be most thoroughly rubbed up with water until it goes into suspension, and great care must be taken to avoid surface films and films upon air bubbles; the inlet of the siphon 
must be kept well away from the settled grit, since the latter is easily disturbed. The upper limit for the medium grade is a fall of 6 inches in two minutes and the lower limit an equal distance in ten minutes; but the latter time could probably with advantage be reduced from ten minutes to five or six, thus removing some of the finest particles. The upper limit of the fine grade is a fall of 6 inches in half an hour and the lower limit 12 inches in eighteen hours; but beyond doubt the grit would be improved by moving both time limits nearer together with an upper limit of one hour and a lower of six to eight hours. The settling process, filtration, and drying are carried out as previously described, remembering that whenever coarse grit is to be eliminated the bottle must be filled to overflowing before introducing the siphon and that the inlet of the siphon should never extend more than half-way to the bottom of the bottle. A large portion of the grit will be too coarse and must be ground before refining. A little grit is mixed with water to form a paste and ground between two glass plates which are then washed in a large pan of water, and the process repeated until the water is very milky; the added glass particles cut rapidly and are not objectionable. The milky water is poured into a large bottle and the coarse grit remaining in the pan saved for grinding. From this milky suspension the two grades of grit are prepared, first eliminating grit too coarse for the medium grade and then that which is too fine; from the latter the fine grade of grit is prepared. On testing the medium grade the largest particles should not measure more than $30 \mu$ in diameter and there should be few below 8, while the average is about $17 \mu$; as previously stated, I advise changing the lower limit from ten minutes to five or six. The fine grade should have no particle over $13 \mu$; the smallest are about $0.5 \mu$, and the average about 4 . This variation is certainly too great, and I strongly advise changing the upper limit from half an hour to one hour, and the lower limit from eighteen hours to six or eight hours.

The general method of using the hones will next be described, followed by additional observations in the case of each hone. The fundamental fact to remember is that the knife should always 
rest with merely its own weight upon the hone; all external pressure, however tempting, should be avoided, and the other source of pressure, namely adhesion, must be as carefully avoided through the proper care of the surface of the home. The strokes employed are the same as in stropping, oblique strokes on alternate sides with the back moving in advance of the edge and the toe in advance of the heel; a handle must not be used nor should any portion of the knife project beyond the edge of the hone. Before each stroke the surface of the hone is lightly roughened up with the palm of the hand; this prevents the grit from packing into a hard layer and maintains a thin film of loose grit on the surface. Such a surface film of loose grit prevents adhesion of the knife to the hone and causes the latter to cut more rapidly. If sticky patches of grit appear, they can often be removed by vigorous rubbing of the entire surface of the hone with the hand so as to mix the oily grit with the dry, but at times the excess of oil must be removed by rubbing off the sticky paste with clean paper, after which fresh grit is applied and smoothed out with the palm. To prevent the formation of gaps through breaking the thin edge, the latter should, except in the case of the fine hone, be kept rounded and smooth. This is accomplished by lightly drawing the edge several times over the ball of the thumb covered with grit; the edge is removed before it can enter the epidermis. The edge is restored by about five double strokes on the hone, and accordingly the dulling should be repeated often, the frequency of the process depending upon the thoroughness with which it has been carried out.

The coarse hone is used when gaps exist, when the facets fail to intersect, or in any condition where much steel has to be removed, and when these defects have disappeared nothing is to be gained by longer use of this hone. The edge should be kept well rounded and smooth as just described, but if any doubt exists as to the intersection of the facets throughout their entire length, this dulling process should be discontinued until the edge along its entire length will cut a coarse hair; thereafter the dulling operation is resumed. Since the coarse hone should cut rapidly, the surface layer of loose grit should be thick, about $1 \mathrm{~mm}$. in 
thickness, and should be brushed off and replaced with fresh grit when it becomes oilyand broken up into finer particles, thus slowing its cutting. Near the end of the operation the excess of grit should be removed, leaving a surface layer of loose grit about $1 / 4 \mathrm{~mm}$. in depth, and this grit should not be replaced by fresh; in this manner the facets will be more nearly plane and the knife in better condition to transfer to the medium hone.

The medium hone is used after the coarse and also to remove gaps which have appeared in the finished edge when these do not exceed a size barely visible to the unaided eye. As in the case of the coarse hone, it should have a surface layer of loose grit lightly roughened up by hand before each stroke. But since at this stage rapid cutting is of less importance than accuracy, this surface layer should be thin, about $\frac{1}{5} \mathrm{~mm}$ : in depth, and since the grit is relatively valuable it should be renewed only when necessary. Upon the gradual intermixture with oil, this grit will form a sticky paste more readily than the coarse grit, and accordingly more attention is necessary to maintain the thin surface film of loose grit so essential to prevent adhesion between the knife and hone. Greater care against contamination is necessary and before using the hone or when it otherwise appears advisable, the surface should be blown off and then lightly brushed off by hand, adding fresh grit when necessary. With the foregoing modifications the procedure is the same as usual, the knife moving obliquely under its own weight alone, back forward and toe in advance of heel, reversing sides and direction after each stroke, the surface grit lightly disturbed before each stroke, and the edge kept rounded and smooth. A medium polish appears on the facets, and the edge if not purposely dulled, as it should be at least at the beginning and the termination of the process, becomes very keen, but shows slight microscopic defects.

The fine hone is used after the medium hone and also before and after employing the knife for cutting. Its fine grit has the tendency to pack into a firm smooth layer which may cause the knife to adhere and its edge to break, and consequently a little fresh grit should be added after about twenty double strokes. 
The fresh grit has the feel of talcum and should be frequently added in small quantities forming above the firm smooth layer of grit a film about as thick as a rather heavy film of talcum. If the hone is thus kept with a surface which has the feel of a film of talcum it will cut relatively fast, and the knife will glide over it with the greatest ease, showing no tendency to adhere. The method of sharpening is as usual, the surface layer being very lightly disturbed before each stroke, and all pressure on the knife as well as all careless manipulation being carefully avoided. This stage of honing should be carried out with deliberation, keeping clearly in mind that by the delicate removal of minute films of steel one is bringing to complete intersection two polished and perfectly plane facets; with such an ideal in mind any position of the knife which allows the facet to rest upon the hone in any manner other than one of light yet perfect contact will be recognized as the result of stupid carelessness in the manipulation of the knife. At first the edge should be kept dull as described, but later of course it must be allowed to reach its maximum keenness. After the imperfections due to the medium hone have been removed, the final finish is given to the edge by allowing the surface grit to pack somewhat, slightly disturbing it after every second double stroke, instead of after every single stroke. This gives an edge which is keener and more highly polished, but the tendency of the knife to adhere must not be allowed to go too far. It is evident that very careful refining of the grit is essential, for the perfection of the finished edge depends upon the nature of the grit.

The resulting edge is very keen, with highly polished facets, and should be used without attempting to improve it on the usual leather strop. Oiled leather without grit will cause the knife to adhere firmly, and if it is not oiled the surface is very apt to have rough places. Such rough places on soft leather will round off the edge, while on hard leather they will cause deep scratches and gaps to an extent almost unbelievable. A great deal of damage is done to knives by stropping, the nature of the injury depending upon whether the surface is sticky, fuzzy, or hard and rough. Even if a strop is hard and smooth it offers a surface upon which 
a piece of lint or a particle of grit will do the maximum damage to the knife.

The edge should be tested by inspection under the microscope and by the manner in which it will cut a hair. Both of these tests are necessary, since in my opinion the keenness of the edge cannot be determined by inspection, which, however, gives most reliable information as to uniformity; while the manner of cutting a hair will give reliable information as to the relative keenness of large portions of the edge, but fails to indicate the extent or even the presence of sharply localized defects. The microscopic inspection is carried out by reflected light under the $16 \mathrm{~mm}$. lens. A firm support, such as a book or a board, is placed on the stage and covered with a piece of clean paper upon which the knife is placed flat with the edge directly facing the light. The knife must not be moved over the paper, but only by moving its support over the stage; the light should always fall directly upon the cutting-facet from in front and above. In testing for keenness one should remember that while a rough edge will cut a coarse hair more readily than a fine hair, the reverse is true of a finely serrated edge, since it cuts a soft delicate hair with ease, but a coarse hard one only with the aid of pressure. An edge finished on the fine hone should throughout its entire length instantly sever a fine hair with extreme suddenness and smoothness. If the edge throughout its length meets this test of keenness and under low power of the microscope reveals no appreciable inequalities it is thoroughly serviceable. In fact, an edge which shows a few very minute gaps is for most purposes usable, since the loose grit tends to polish and round off and thus lessen such slight defects, an advantage especially apparent when the knife is used in the oblique position which permits such rounded and polished depressions to remain practically inactive.

A knowledge of the principles which underlie the process of sharpening is essential to success, and I have attempted to make their consideration sufficiently general to apply to all methods employing a plane hone. Since the principles and methods have been discussed somewhat at length, the impression may result

THE ANATOMICAI RECORD, VOL. 24, NO. 3 
that the method described must of necessity be complicated. But the complexity lies in the operation of a large hone with partially fixed grit, a hone which through its lack of simplicity of operation automatically removes many sources of danger and thus permits the operator to attain success with certainty and with a minimum of skill. While the requisite skill has been greatly reduced, the delicacy of the edge is such that no method will assure good results in the absence of intelligence and care. Accordingly, it has appeared desirable to present a thorough discussion of the principles, aims, procedures, and errors, since their appreciation must always form the basis of intelligent effort. 